\title{
The Odontogenic Keratocyst Detected Accidentally With Radiological Features
}

\author{
Erwin Setyawan ${ }^{1, *}$ \\ ${ }^{1}$ School of Dentistry, Faculty of Medicine and Health Sciences, Universitas Muhammadiyah Yogyakarta, Yogyakarta, \\ Indonesia \\ "Coressponding author. Email: erwinsetyawan@ fkik.umy.ac.id
}

\begin{abstract}
Odontogenic Keratocyst (OKC) usually appears asymptomatically; thus, it is likely to be found incidentally. World Health Organization (WHO) classifies OKC into tumors as they have similar characteristics. Although it is classified as a non-malignant tumor, odontogenic keratocyst can occur repeatedly. It tends to affect the surrounding tissue when the tumor gets bigger. This radiographic image of the OKC has somewhat specific features. The aims of the research is to identify the OKC in a panoramic radiograph. Case: a 24-year old woman complained that She frequently felt pain in her lower left molar; thus, it sometimes felt uncomfortable to chew food. The intraoral examination of 38 showed percussions $(+)$ and there were no abnormalities in the mucosa around the tooth. The radiographic examination showed the radiolucent lesion in the apical tooth 38 that extended towards the anteroposterior with a diameter of $\pm 30 \mathrm{~mm}$. Discussion: OKC can be derived from the proliferation of oral epithelial basal cells, having the innate growth potential, consistent with a benign tumor. In this case, OKC goes along with pain in the involved tooth. The radiographic features of OKC shows unilocular radiolucent lesion with a well-defined and cortical border like a 'scalloped outline'. Conclusion: OKC sometimes appears to be asymptomatic; thus, sufficient knowledge of OKC radiographs can help identify the lesion early.
\end{abstract}

Keywords: keratocystic odontogenic tumor, KOT, odontogenic keratocyst, OKC, scallop

\section{INTRODUCTION}

Odontogenic keratocyst (OKC) was firstly introduced by Philipsen [1], It is a non-inflammatory odontogenic cyst arising from the dental lamina. OKC can occur during the immature tooth formation process, namely at the bell stage [2]. Eleven percent OKC cases of all cysts occur in the jaws, most of which occur in the mandible [3]. OKC is mostly found at the age of 10-40 years old, and the incidence more commonly happens in males than females with a ratio of $2: 1$ [4]

Odontogenic keratocyst (OKC) is a type of cystic lesion that was previously classified into a cyst type. In 2005, it was reclassified by WHO into the odontogenic tumor category under the name Keratocystic Odontogenic Tumor (KOT or $\mathrm{KCOT}$ ). According to WHO, the histological classification of KCOT odontogenic tumors has been defined as a benign uni or multicystic intraosseous tumor originated from odontogenic, with a distinctive layer of parakeratosis stratified squamous epithelium and a potentially aggressive, infiltrative behavior [5]. The characteristics of the OKC epithelial layer resemble that of the tumor type. The difference in the growth of mechanisms provides different radiographic images of the cyst [6]. In 2017, WHO reclassified KCOT into cysts under the name odontogenic keratocyst as there was not enough evidence to support the neoplastic origin [7].

Small odontogenic keratocysts are usually asymptomatic and only visible on radiographs. In larger lesions, they usually causes pain, swelling, and a fistula. These lesions can also result in jaws deformity. The tendency of recurrence in various treatments is $2.5-62.5 \%$ [8]. Based on the characteristics of the $\mathrm{OKC}$, it is necessary to have sufficient knowledge regarding the clinical and radiographic features; thus, these lesions can be identified early on even though they originate from accidental radiographs.

\section{CASE}

A 24-year-old woman complained that her lower left tooth often had pain, so it was sometimes uncomfortable to use for chewing. The extraoral examination did not show any abnormalities. Intraoral examination of 38 showed percussions (+) without any abnormalities in the mucosa around the tooth. There was no swelling on it. The dentist prescribed antibiotics and analgesics related to the patient's condition. A week after the drug administration, the pain 
remained. To better identify the condition of tooth 38 and its surrounding tissue, it was advisable to do a radiographic examination; thus, the treatment plan that would be given related to the patient's complaint could be undoubtedly determined.



Figure 1. Panoramic radiograph: showing lesion in the apical region of tooth 37 and 38

Based on the panoramic radiograph, there was a radiolucent lesion in the apical region of teeth 37 and 38 . The lesion was unilocular with an anteroposterior diameter of approximately $3 \mathrm{~cm}$. It was a well-defined border that looked a bit sclerotic corticated as a curved edge. The mandibular canal was pushed inferiorly by the lesion. The radio diagnosis suspect of the lesion revealed odontogenic keratocyst with a differential diagnosis of ameloblastoma.

Based on the diagnosis, the entire lesion of the tissue was taken, and some part of the tissue was sent to the anatomical pathology department. The result of the histopathological examination showed the layer of the squamous epithelial complex on the wall of the lesion. The lumen of the lesion contained large keratin masses, and there were no signs of malignancy on it. Based on the histopathological examination, it can be concluded that there was odontogenic keratocyst.

\section{DISCUSSION}

The patient who experienced this case was 24 years old. She was in the range of 20-30 years old, the age which is likely to experience OKC [9]. Odontogenic keratocyst, in this case, appeared along with frequent pain when the patient chewed food in the area of tooth 38. The pain provided specific suspicion of an abnormality in the area of tooth 38 ; thus, a radiograph examination was required to help provide information on the conditions. OKC cases are usually asymptomatic or appear to have slight swelling. It goes along with pain due to a secondary infection [6]. OKC is usually found incidentally on routine radiographic examinations [4].

Radiographs with panoramic techniques can be used to assist in the analysis of the location of the lesion, the lesion boundary, and the extent of the lesion [7]. The patient's panoramic radiographs showed apparent radiolucent lesions with well-defined and corticated border. It is in line with the study conducted by other researchers, stating that, in the radiograph examination, unilocular $\mathrm{OKC}$ is a depiction of unilocular cystic radiolucent lesion that is a well-defined border and is surrounded by smooth radiopaque boundaries or curved-arch as "scalloped outline"[6,11]. These cortical boundaries are sometimes invisible due to a secondary infection [6].

Due to the resorption of trabecular bone in the lesion shown in the radiolucent, keratinized tissue sometimes does not make a change in the internal density of the lesion [6]. If the internal lesion looks rather vague, it is possibly due to the lumen filled with keratin [11].

The OKC growth, in this case, has not expanded the mandibular cortex as the position of the lesion is in the ramus of the mandible. If the location of the lesion is at the top of the ramus of the mandible, it is possible to expand the cortical layer. It can occur due to a delay in recognizing the lesion. Thus, the lesion can be identified when it gets larger and develops the cortical layer [6]. Most of the time, the growth of the lesion goes towards the anteroposterior so that the diameter of the lesion towards the mesial-distal looks more significant than the superior-inferior direction [12].

The differential diagnosis of $\mathrm{OKC}$ includes ameloblastoma as both have similar depictions. The ameloblastoma also has a scalloped margin, but the expansion of the ameloblastoma is faster [6]. The differences of the radiolucency in the internal lesion can also be utilized to distinguish these two lesions by using computed tomography (CT) radiographs with more radiolucent internal OKC (lower density on CT) as a result. Ameloblastoma mostly also results in root resorption of the involved teeth while it rarely occurs in the OKC case [10].

\section{CONCLUSION}

Small odontogenic keratocysts are usually asymptomatic and identified incidentally by a routine radiograph examination. More detailed knowledge about $\mathrm{OKC}$ radiographs is required to identify the lesion earlier.

\section{REFERENCES}

[1] Philipsen HP. Keratocystic odontogenic tumor. In: Barnes L, Eveseon JW, Reichert P, Sidransky D (eds). Pathology and genetics of head and neck tumors. Lyon: World Health Organization. IARC Press, 2005, pp 305307.

[2] Pogrel MA. The history of the odontogenic keratocyst. Oral Maxillofac Surg Clin North Am. 2003;15(3):311315.

[3] Maurette PE, Jorge J, de Moraes M. Conservative treatment protocol of odontogenic keratocyst: a preliminary study. J Oral Maxillofac Surg. 2006;64(3):379-383. 
[4] Roa I, Robles P. Keratocystic odontogenic tumor: Clinicopathological aspects and treatment. Journal of Oral Research. 2014;3:249-256.

[5] MacDonald-Jankowski DS, Li TK. Keratocystic odontogenic tumour in a Hong Kong community: the clinical and radiological features. Dentomaxillofacial Radiology. 2010;39(3):167-175.

[6] White SC, Pharoah, MJ. Oral Radiology Principles and Interpretation $7^{\text {th }}$ Ed. Philadelphia: Mosby Co; 2014.

[7] Alves DBM, Tuji FM, Alves FA, et al. Evaluation of mandibular odontogenic keratocyst and ameloblastoma by panoramic radiograph and computed tomography. Dentomaxillofacial Radiology. 2018;47(7):20170288.

[8] Pogrel MA, Kahnberg KE, Andersson L. Essentials of Oral and Maxillofacial Surgery. West Sussex: Wiley Blackwell; 2014.
[9] Raouâa B, Samèh S, Dorsaf T, Rahma A, Jamil S. Recurrence challenge in odontogenic keratocyst variants, two clinical cases. Med Buccale Chir Buccale. 2014;20(3):203-208.

[10] Ariji Y, Morita M, Katsumata A, et al. Imaging features contributing to the diagnosis of ameloblastomas and keratocystic odontogenic tumors: logistic regression analysis. Dentomaxillofacial Radiology. 2011;40(3):133-140.

[11] K, Li X, Wang H, et al. Correlation between imaging features and epithelial cell proliferation in keratocystic odontogenic tumor. Dentomaxillofacial Radiology. 2010;39(6):368-374.

[12] Tonietto L, Borges HOI, Martins CAM, Silva DN, Sant'Ana Filho M. Enucleation and liquid nitrogen cryotherapy in the treatment of keratocystic odontogenic tumors: a case series. J Oral Maxillofac Surg. 2011;69(6):e112-117. 